\title{
Relationship of Cytokeratin 20 and CD44 Protein Expression with WHO/ISUP Grade in pTa and pT1 Papillary Urothelial Neoplasia
}

Sangeeta Desai, So Dug Lim, Rafael E. Jimenez, Thomas Chun, Thomas E. Keane, Jesse K. McKenney, Angel Zavala-Pompa, Cynthia Cohen, Robert H. Young, Mahul B. Amin

Departments of Pathology and Laboratory Medicine (SD, SDL, REJ, JKM, AZ-P, CC, MBA) and Urology (TC, TK, MBA), Emory University School of Medicine, Atlanta, Georgia and Massachusetts General Hospital and Harvard Medical School (RHY), Boston, Massachusetts

The aim of this study was to assess the relationship of immunoreactivity of cytokeratin 20 (CK20) and CD44 across the spectrum of urothelial neoplasia using the WHO/ISUP consensus classification. A total of 120 papillary urothelial pTa and pT1 tumors (8 papillomas, 8 neoplasms of low malignant potential, and 42 low-grade and 62 high-grade carcinomas) were immunostained by using CK20 and CD44 antibodies. The relationships of tumor grade, pathologic stage, recurrences, and progression in stage with CK20 and CD44 immunoreactivity were assessed. WHO/ISUP grade correlated with tumor stage $(P<0.005)$, recurrence $(P=0.02)$, and progression in stage $(P=0.031)$. Normal urothelium showed CK20 immunoreactivity restricted to a few umbrella cells. Expression of CD44 in normal urothelium was restricted to the basal cell layer. Loss of CD44 immunoreactivity and increasing CK20 positivity were significantly associated with increasing tumor grade and stage $(P<0.005)$. An inverse relationship was observed in the staining patterns of CK20 and CD44 within individual cases, as well as in the aggregate data, with $79.2 \%$ of tumors with CD44 loss showing CK20 positivity $(P<$ 0.001). In conclusion, CK20 and CD44 immunoreactivity are significantly related to the WHO/ISUP grade and to each other, and our data suggest their potential combined utility in predicting biologic behavior in patients with papillary urothelial pTa and pT1 neoplasms.

Copyright () 2000 by The United States and Canadian Academy of Pathology, Inc.

VOL. 13, NO. 12, P. 1315, 2000 Printed in the U.S.A.

Date of acceptance:

Address reprint requests to: Mahul B. Amin, M.D., Emory University Hospital G-167, 1364 Clifton Road NE, Atlanta, GA 30322; e-mail: mahul_amin@emory.org; fax: 404-712-0148.
KEY WORDS: Urinary bladder, Papillary neoplasia, Transitional cell carcinoma, WHO/ISUP classification, Pathologic stage, CK20, CD44, Prognosis.

Mod Pathol 2000;13(12):1315-1323

More than $50 \%$ of patients with papillary urothelial tumors not invasive into the muscularis propria (pTa and pT1) have recurrences (or new occurrences), and 10 to $15 \%$ of these will progress to muscularis propria invasive cancer $(1,2)$. Therefore, the identification of prognostic markers in papillary urothelial tumors (pTa and pT1) that could identify subsets of patients with a high probability of recurrence or progression would be important in patient management.

Although tumor grade and stage have been shown to have a strong correlation with tumor recurrence and progression to invasive disease in bladder cancer $(1,3-6)$, the recently proposed (in 1998) World Health Organization and International Society for Urological Pathology (WHO/ISUP) system (7), based entirely on morphological criteria, has not yet been compared with objective markers that may correlate with disease outcome.

A large number of markers have been extensively investigated as potential predictors of bladder tumor recurrence, progression, or response to therapy (8). A selective few include altered oncogene and tumor suppressor genes $(9-11)$, growth factors and growth factor receptors (12-15), intermediate filament expression (8), and cell proliferation markers $(8,11)$. Recently, CD44 and cytokeratin 20 (CK20) expression have been studied in bladder carcinoma, and the data suggest that they may be useful in the diagnosis of urothelial carcinoma on the basis of tumor immunoreactivity (IR; 16-21) or by using reverse transcription-polymerase chain reaction in urine cytology (22-24).

CK20 belongs to the epithelial subgroup of cytoskeleton-associated intermediate filaments (25) 
and has restricted expression in the gastrointestinal and urinary tracts, in Merkel cells, and in their respective neoplasms $(25,26)$. It has been suggested that CK20 may be an important tool for detecting and identifying these types of cancer and their metastases, either by immunohistochemistry or by reverse transcription-polymerase chain reaction analysis.

CD44 encompasses a family of polymorphic transmembrane glycoproteins involved in cellsurface binding to hyaluronidate collagen (27), fibronectin, and ankyrin $(28,29)$. It has been implicated in cell-cell adhesion and cell matrix cytoskeletal interactions (30); lymphocyte activation, recirculation, and homing $(31,32)$; and tumor invasion (33). Alternative splicings of the CD44 standard form gene, occurring between Exons 5 and 16, gives rise to many CD44 isoforms (CD44v). There has been interest in the expression of CD44 as a marker of tumor aggressiveness and metastatic potential in human breast cancer $(30,34)$, gastric carcinoma (35), and colonic carcinoma (36, 37), as well as in urothelial carcinoma $(16,18)$. It has been suggested that CD44 not only functions as an adhesion protein but may also play an important role in cytoskeleton-mediated cellular signals, suggesting a possible interaction between CD44 and CK20 at the cellular level. The interrelationship between CD44 and CK20 IR in urothelial neoplasms has not been previously studied.

In this study, we evaluated the relationship of CD44 and CK20 IR in noninvasive (pTa) and lamina propria-invasive (pT1) papillary urothelial neoplasia, as graded by the WHO/ISUP consensus classification. We also studied the association between conventional pathologic parameters and clinical outcome, including tumor recurrence and progression in stage.

\section{MATERIALS AND METHODS}

\section{Clinicopathological Data}

All cases of pTa and pT1 papillary urothelial tumors accessioned at Emory University Hospital between 1979 and 1995 were studied. Additionally, eight urothelial papillomas retrieved from the consultation files of two of the authors (MBA and RHY) were added to study the entire spectrum of grades of urothelial papillary neoplasia. Pathologic material included bladder biopsies, transurethral resections of bladder tumor, and cystectomies.

All available bladder tumors from a total of 120 patients were classified according to the 1998 WHO/ISUP grading system (7) and staged according to the 1997 TNM staging system (38). Because grade variation exists in papillary urothelial neoplasms, tumors were graded by the worst grade present. Ten cases of high-grade invasive urothelial carcinomas, as well as 15 sections of ureteric margins from radical nephrectomy specimens performed for renal cell carcinoma, were also added for comparison of immunostaining patterns.

Follow-up data, including grade progression, tumor recurrence, muscle invasion, distant metastasis, and death from disease were obtained from medical records. Recurrence was defined as a new tumor appearing at any site in the bladder at least 3 months after initial resection. Progression in stage was defined as development of lamina propria invasion (for pTa tumors) or muscularis propria invasion (for pTa and pT1 tumors), metastasis, or death from disease. Grade progression was defined as the subsequent development of a tumor with a higher grade. Follow-up data were available in 106 patients for tumor recurrence and in 98 patients for progression in stage.

\section{Immunohistochemistry}

In every case, sections from the first specimen were studied by immunohistochemistry. Fivemicrometer-thick paraffin tissue sections of each case were deparaffinized and rehydrated, then steamed in citrate buffer ( $\mathrm{pH} \mathrm{6)}$ ) for 20 minutes and cooled for 10 minutes. The tissue was then exposed to $3 \%$ hydrogen peroxide for 5 minutes, to primary antibody for 25 minutes, to biotinylated secondary linking antibody for 25 minutes, to streptavidin enzyme complex for 25 minutes, to diaminobenzidine as chromogen for 5 minutes, and to hematoxylin counterstained for 1 minute.

The antibodies used were anti-human mouse IgG CD44 monoclonal antibody (Pharmingen, San Diego, CA) at a dilution of 1:640 and anti-human mouse IgG CK20 monoclonal antibody (DAKO, Glostrup, Denmark) at a dilution of 1:20. Immunohistochemistry was performed by using automated immunostainer, Techmate 1000 (Biotek Solutions, Santa Barbara, CA).

These incubations were performed at room temperature, and between incubations, sections were washed with Tris-buffered saline buffer. Both primary antibodies were detected by using an avidinbiotinylated enzyme complex kit.

\section{Immunohistochemical Analysis}

The urothelium of the urinary bladder and of many papillary tumors consists of basal, intermediate, and superficial umbrella cell layers. The sections were evaluated for the degree and pattern of IR for both antibodies in the different cell layers. For tumors showing grade heterogeneity, IR was analyzed predominantly in areas corresponding with the highest grade. A pilot review of cases 
yielded the following IR patterns that were then used as a basis for a more detailed evaluation of the cases.

CD44 was strongly expressed on the plasma membranes of basal cells in normal urothelium (designated normal basal staining pattern), as well as in stromal cells, including fibroblasts, endothelial cells, myocytes, and lymphocytes, consistent with previous reports (16, 17, 39; Fig. 1A). The suprabasal intermediate cells of the urothelial epithelium were negative. However, most papillary neoplasms showed CD44-positive IR in the suprabasal intermediate cells, as well as in the basal cells, but not in the superficial cells (designated accentuated staining pattern). Further, some tumors displayed focal loss of CD44 expression, either in the background of predominantly basal or accentuated staining pattern. Thus, CD44 IR was classified into four reproducible patterns: (1) normal basal pattern, showing staining of only basal cells, as in the normal urothelium; (2) accentuated pattern, showing staining throughout most layers of the epithelium; (3) loss-N pattern, showing partial or total loss of otherwise normal basal cell staining; and (4) loss-Ac pattern, showing focal loss of staining in an otherwise accentuated pattern. For statistical analysis, the two former and the two latter patterns, respectively, were categorized as the CD44 N/Ac and CD44 Loss groups.

In the normal urothelium, CK20 IR was limited to occasional strong staining of umbrella cells (Fig. 1B). Basal and intermediate urothelial cells were always negative. In contrast, CK20 IR was variable in the neoplastic urothelium. Thus, CK20 IR was classified in four categories: (1) normal pattern, restricted to staining of only superficial cells as in the normal urothelium; (2) absent staining; (3) focal pattern, in which less than $10 \%$ of tumor cells stained; and (4) diffuse pattern, in which more than $10 \%$ of tumor cells were positive.

For statistical analysis, the first two patterns and the last two patterns were categorized as the CK20negative group and the CK20-positive group, respectively.

\section{Statistics}

Statistical analysis was done with SPSS software, Version 7.0, for Windows 98. The relationship between the pathologic data, including WHO/ISUP grade and pathologic stage, patterns of CK20 and CD44 expression, and clinical outcome, was analyzed by using the $\chi^{2}$ test. All $P$ values were two tailed, and a $P$ value $<0.05$ was considered significant.

\section{RESULTS}

The clinical data, WHO/ISUP grade, and pathologic stage of 120 patients with papillary urothelial neoplasms are summarized in Table 1. Of 120 tumors, eight tumors were papillomas, eight were papillary urothelial neoplasms of low malignant potential, 42 were low-grade, and 62 were high-grade papillary urothelial carcinomas.

The mean age of the patients with papilloma and papillary urothelial neoplasms of low malignant potential was lower than that of those with low-grade papillary carcinoma and with high-grade papillary carcinoma $(P<0.005)$. There was no difference in the mean age of patients with low-grade papillary carcinoma and high-grade papillary carcinoma $(P$ $=0.618$ ). During a mean follow-up of 7.4 years (median, 6 years), $54.7 \%$ of tumors recurred, and $17.3 \%$ of tumors progressed in stage. WHO/ISUP grade correlated with tumor stage and recurrence $(P<0.005, P=0.02$, respectively) and with tumor progression in stage when analyzed as papilloma and papillary urothelial neoplasm of low malignant potential versus low-grade papillary carcinoma versus high-grade papillary carcinoma $(P=0.031)$ (Table 1). No lamina propria or muscularis propria invasion, metastasis, or death from disease was seen in patients initially diagnosed with papilloma or papillary urothelial neoplasm of low malignant potential.

There was a significant correlation between CD44 IR and $\mathrm{WHO} /$ ISUP grade (Table 2). The dominant pattern of CD44 expression within each grade was
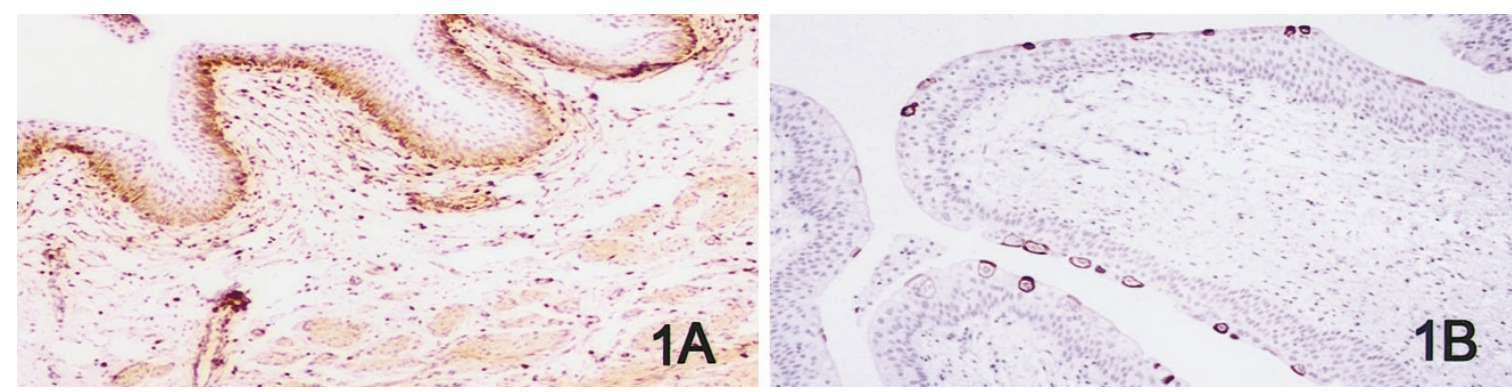

FIGURE 1. Normal urothelium (normal ureter). A, CD44 immunoreactivity is noted as strong membrane staining of basal cells. B, CK20 immunoreactivity is limited to strong staining of occasional superficial cell, less than $10 \%$. 
TABLE 1. Comparison of WHO/ISUP Grade and Stage with Clinical Data in 120 Papillary Urothelial pTa and pT1 Urothelial Neoplasms

\begin{tabular}{|c|c|c|c|c|c|c|}
\hline WHO/ISUP grade & Pap & LMP & LG & HG & Total & $P$-value \\
\hline \multicolumn{7}{|l|}{ Stage } \\
\hline pTa & $100 \%(8)$ & $100 \%(8)$ & $97.6 \%(41)$ & $50 \%(31)$ & $73.3 \%(88)$ & $<0.005$ \\
\hline pT1 & 0 & 0 & $2.4 \%(1)$ & $50 \%(31)$ & $26.7 \%(32)$ & \\
\hline Total \% (no. of cases) & $6.7 \%(8)$ & $6.7 \%(8)$ & $35 \%(42)$ & $51.7 \%(62)$ & $100 \%(120)$ & \\
\hline Mean age (yrs)* & 42.8 & 53.4 & 64.5 & 65.6 & 62.9 & $<0.005$ \\
\hline Sex (M:F) ratio & $3: 1$ & $1: 1.7$ & 7.4:1 & 7.9:1 & 5.3:1 & \\
\hline Recurrence $^{* *}$ & 0 & $33.3 \%$ & $64.1 \%$ & $56.4 \%$ & $54.7 \%$ & 0.02 \\
\hline Progression in stage st** $^{* *}$ & 0 & 0 & $10.5 \%$ & $27.1 \%$ & $17.3 \%$ & 0.031 \\
\hline pTa to pT1 & 0 & 0 & $2.6 \%$ & $8.3 \%$ & $8.6 \%$ & \\
\hline Muscle invasion & 0 & 0 & $5.3 \%$ & $6.3 \%$ & $5.1 \%$ & \\
\hline Metastasis & 0 & 0 & $5.3 \%$ & $8.3 \%$ & $6.1 \%$ & \\
\hline Death from disease & 0 & 0 & $5.3 \%$ & $16.7 \%$ & $10.2 \%$ & \\
\hline
\end{tabular}

Pap, papilloma; LMP, papillary urothelial neoplasm of low malignant potential; LG, low-grade papillary urothelial carcinoma; HG, high-grade papillary urothelial carcinoma.

* Analyzed as Pap and LMP vs. LG and HG or Pap, LMP and LG vs. HG.

** Data available for tumor recurrence in 106 of 120 cases.

*** Data available for tumor progression in 98 of 120 cases. Data analyzed as Pap and LMP vs. LG vs. HG.

the normal pattern $(75 \%)$ in papilloma, the accentuated pattern $(50 \%)$ in papillary urothelial neoplasm of low malignant potential, the accentuated pattern (38.1\%) in low-grade papillary carcinoma, and the loss-N pattern (61.3\%) in high-grade papillary carcinoma $(P<0.001)$, respectively. Further, $83.9 \%$ of high-grade papillary carcinoma, $47.6 \%$ of low-grade papillary carcinoma, $37.5 \%$ of papillary urothelial neoplasm of low malignant potential, and $25 \%$ of papilloma showed loss patterns (the loss- $\mathrm{N}$ and loss-Ac patterns) of CD44 expression ( $P$ $<0.001$ ). Thus, a progressive loss of CD44 IR was associated with an increasing tumor grade (Table 2 , Figs. 2A, 2B, 3A, 3B, 4A, 4B, 5A, 5B).

There was increasing CK20 expression as the WHO/ISUP grade increased (Table 3 ). When the patterns were grouped as mentioned in the Materials and Methods section, $69.4 \%$ of the cases with high-grade papillary carcinoma were CK20 positive, compared with less than $45 \%$ of those with other grades $(P=0.001)$. Thus, a higher frequency of CK20 positivity was observed with increasing WHO/ISUP grade (Table 3, Figs. 2A, 2C, 3A, 3C, 4A, 4C, 5A, 5C).

TABLE 2. Comparison of WHO/ISUP Grade and Stage with the Expression Pattern of CD44 Protein in Papillary Urothelial pTa and pT1 Neoplasia $(n=120)$

\begin{tabular}{llllll}
\hline & CD44 N & CD44 Ac & CD44 loss-N & CD44 loss-Ac & $P$-value \\
\hline Pap & $75 \%$ & 0 & $25 \%$ & 0 & \\
LMP & $12.5 \%$ & $50 \%$ & $37.5 \%$ & 0 & $<0.001$ \\
LG & $14.3 \%$ & $38.1 \%$ & $28.6 \%$ & $19 \%$ & \\
HG & $3.2 \%$ & $12.9 \%$ & $61.3 \%$ & $22.6 \%$ & \\
& & & & & \\
Stage & & & & & \\
pTa & $17 \%$ & $29.5 \%$ & $38.6 \%$ & $14.8 \%$ & 0.001 \\
pT1 & 0 & $6.3 \%$ & $65.6 \%$ & $28.1 \%$ & \\
\hline
\end{tabular}

N, Normal pattern; Ac, Accentuated pattern.

For statistical analysis, CD44 N and CD44 Ac were categorized as "CD44 N/Ac" group, and CD44 loss-N and CD44 loss-Ac were categorized to "CD44 Loss" group.
Patterns of CD44 or CK20 expression also showed a statistically significant association with pathologic stage. Ninety-three percent of pT1 tumors showed the loss-N or loss-Ac patterns of CD44 IR, compared with $53.4 \%$ of pTa tumors $(P=0.001$, Table 2). Seventy-five percent of pT1 tumors were CK20positive, compared with $45.4 \%$ of pTa tumors $(P=$ 0.003 , Table 3).

Although $63.2 \%$ of CK20-positive tumors recurred, compared with $44.9 \%$ in the CK20-negative group, this association did not reach statistical significance ( $P=0.06$, Table 4$)$. Similarly, CD44 IR did not show statistically significant correlation with tumor recurrence or stage progression. Grade progression was seen in only three cases (all low-grade papillary carcinomas) and hence was not statistically analyzed with respect to other findings.

We observed a significant inverse relationship in the staining patterns of CD44 and CK20 IR; this was strikingly evident in corresponding areas of some tumors. Areas within a tumor that were CD44 accentuated were usually CK20 negative and vice versa (Fig. 6A-D). This inverse relationship was evident in the aggregate data as well, with $79.2 \%$ of tumors with CD44 loss showing CK20 positivity ( $P$ $<0.001$, Table 5).

Of the 10 high-grade invasive urothelial carcinomas, $40 \%$ showed the focal pattern of CK20 expression, and the remaining $60 \%$ showed a diffuse pattern. Fifty percent of invasive carcinomas showed the loss-N pattern, and 50\% showed the loss-Ac pattern of CD44 expression.

\section{DISCUSSION}

Patients with noninvasive and lamina propriainvasive papillary urothelial neoplasms are most often treated conservatively and have a protracted 

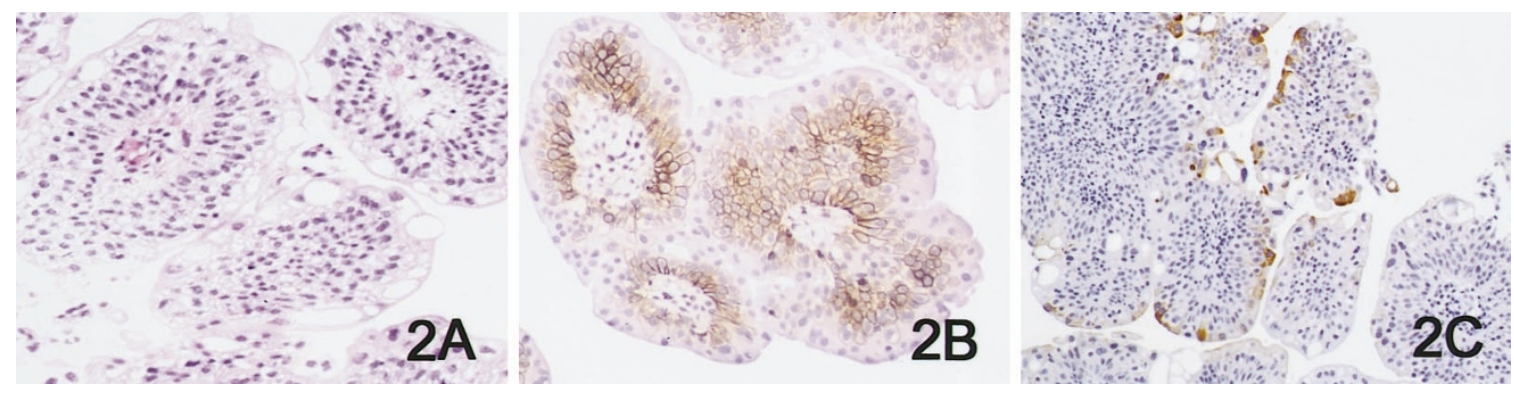

FIGURE 2. Urothelial papilloma. A, hematoxylin and eosin staining. B, CD44 immunoreactivity: basal cell staining. C, CK20 immunoreactivity in superficial cells only, as in normal urothelium.
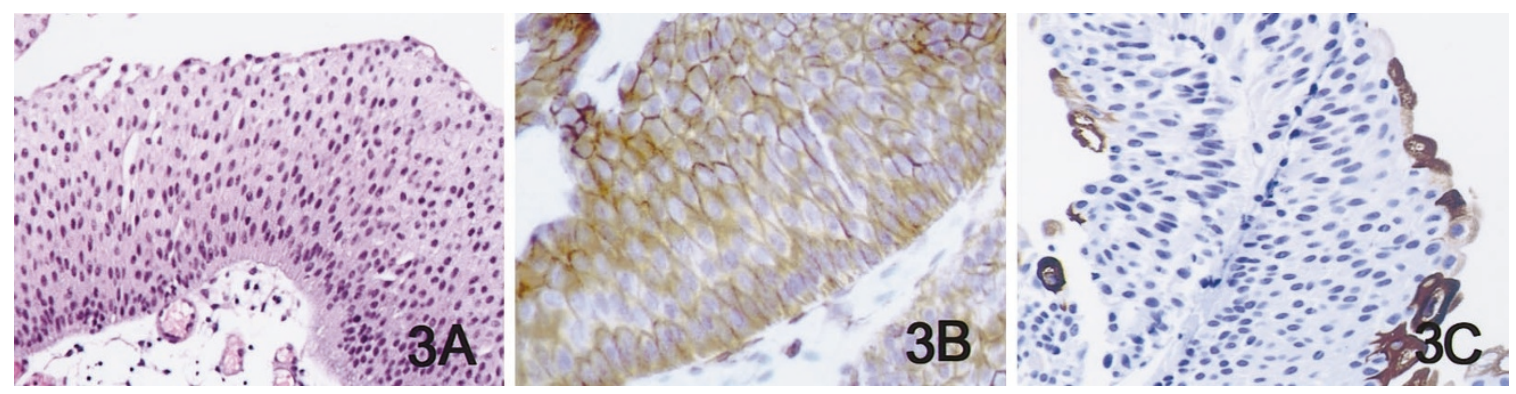

FIGURE 3. Papillary urothelial neoplasm of low malignant potential. A, hematoxylin and eosin staining. B, Accentuated CD44 immunoreactivity throughout all layers of the urothelium. C, CK20 immunoreactivity in a few superficial cells.
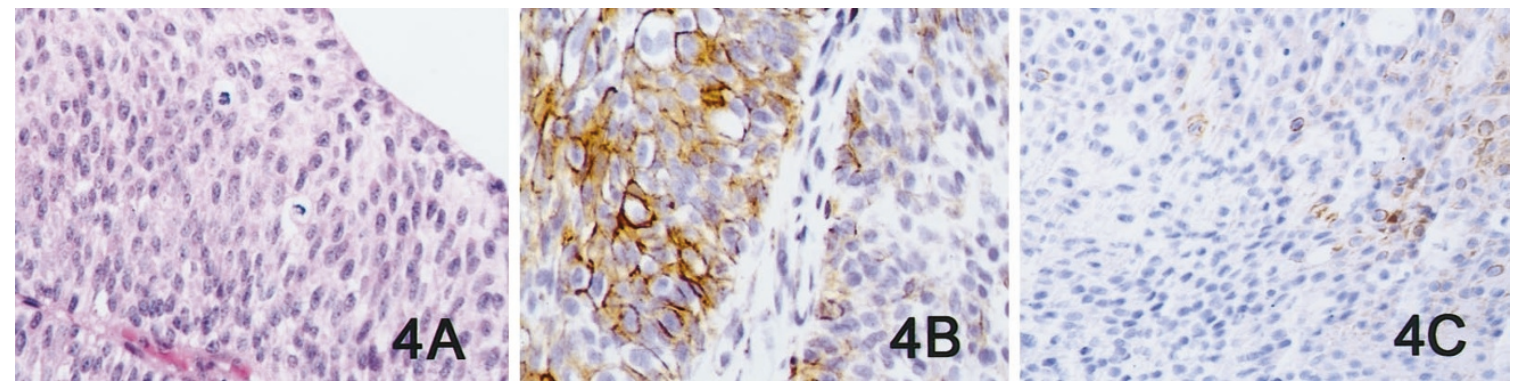

FIGURE 4. Papillary urothelial carcinoma, low grade. A, Hematoxylin and eosin staining. B, Patchy loss of CD44 immunoreactivity in a background of accentuated staining (CD44 loss-Ac). C, Focal CK20 immunoreactivity.

clinical course that requires close clinical follow-up. It is thus useful to identify reliable prognostic parameters that would stratify patients at a higher risk of recurrence and/or progression of the disease.

Our study adds to the emerging clinicopathologic information on papillary bladder neoplasia classified in accordance with the recently proposed WHO/ISUP system (7). The age of patients in our study was statistically different between patients with papilloma and papillary urothelial neoplasm of low malignant potential and patients with papillary carcinoma (low grade and high grade). Holmang et al. (40) have similarly shown statistical difference in age between patients with papillary urothelial neoplasm of low malignant potential versus low-grade papillary carcinoma. Papilloma has a low biologic potential, with recurrences in $0-8 \%$ patients, grade progression in $0-1.9 \%$ patients, and no stage progression (data from Cheng et al. (41) and current series). Recurrence has been reported in 27 to $35 \%$ of patients with papillary urothelial neoplasm of low malignant potential $(40,42)(33 \%$ in current series), whereas 0 to $4 \%(40,42,43)(0 \%$ in current series) of these tumors have stage progression. In contrast, the reported recurrence and stage progression for low-grade papillary carcinoma are $71 \%$ (40) $(64.1 \%$ in current series), and $2.4-12 \%$, respectively $(40,43)(10.5 \%$ in current series). These emerging data suggest that the different WHO/ISUP grades identify clinically and biologically distinct lesions within the spectrum of papillary urothelial neoplasia. Small discrepancies among the reported series are likely explained by differences in the populations studied. For example, in the current series, the relatively low number of tumors in the papillary urothelial neoplasm of 


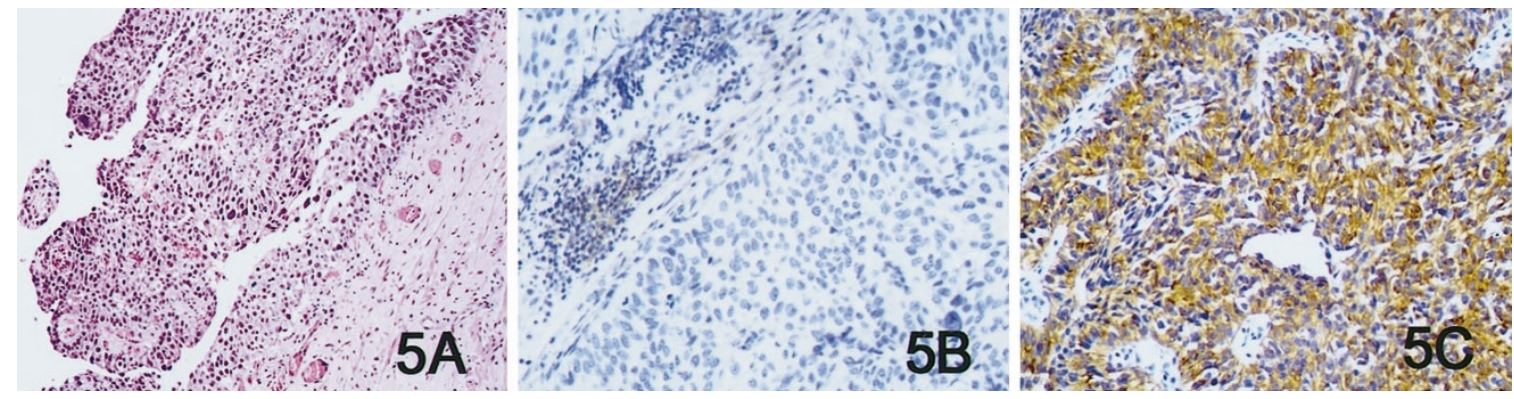

FIGURE 5. Papillary urothelial carcinoma, high grade. A, Hematoxylin and eosin staining. B, Loss-N of CD44 immunoreactivity. C, Diffuse and strong CK20 immunoreactivity.

TABLE 3. Comparison of WHO/ISUP Grade and Stage with the Expression Pattern of CK20 Protein in Papillary Urothelial pTa and pT1 Neoplasia $(n=120)$

\begin{tabular}{lccccc}
\hline & $\begin{array}{c}\text { CK20 } \\
\text { normal }\end{array}$ & $\begin{array}{c}\text { CK20 } \\
\text { absent }\end{array}$ & $\begin{array}{c}\text { CK20 } \\
\text { focal }\end{array}$ & $\begin{array}{c}\text { CK20 } \\
\text { diffuse }\end{array}$ & $P$-value \\
\hline Pap & $87.5 \%$ & 0 & 0 & $12.5 \%$ & \\
LMP & $75 \%$ & 0 & 0 & $25 \%$ & 0.001 \\
LG & $45.2 \%$ & $11.9 \%$ & $19 \%$ & $23.8 \%$ & \\
HG & $17.7 \%$ & $12.9 \%$ & $22.6 \%$ & $46.8 \%$ & \\
& & & & & \\
Stage & & & & & \\
pTa & $46.6 \%$ & $8 \%$ & $13.6 \%$ & $31.8 \%$ & 0.003 \\
pT1 & $6.2 \%$ & $18.8 \%$ & $31.3 \%$ & $43.7 \%$ & \\
\hline
\end{tabular}

For statistical analysis, the first two patterns and the last two were categorized as "CK20 negative" and "CK20 positive" group, respectively.

low malignant potential may reflect an institutional referral bias for higher grade and higher stage tumors.

Our data showed that the dominant pattern of CD44 expression in papilloma, papillary urothelial neoplasm of low malignant potential, or low-grade papillary carcinoma was normal or accentuated pattern. Alho and Underhill (44) have shown that CD44 is expressed on a variety of different epithelia in vivo but that it is particularly noted in rapidly proliferating cells, which may have a crucial role in epithelial maturation. We have also observed an accentuated staining pattern of CD44 IR observed in reactive (45) and highly proliferative urothelium, associated with previous therapy (45). Sugino et al. (46) reported that overexpression of CD44 is observed in the initial stages of bladder and other cancers.

We found a statistically significant progressive loss of CD44 IR with increasing grade and stage in pTa and pT1 papillary urothelial neoplasms. Similar findings related to CD44 IR correlation with WHO grade have been found by others (39). Although in our series, CD44 did not predict recurrence or stage progression, others have shown a significant association between CD44 IR and clinical outcome (17, 18). Lipponen et al. (17) documented that the high expression of CD44v6 in bladder carcinoma was independently related to favorable outcome in muscle invasive tumors (however, loss of CD44v6 IR was not studied). In pTa and pT1 tumors, the authors found that the high intensity of CD44s expression was an independent prognostic factor of unfavorable prognosis by multivariate survival analysis. Toma et al. (18) reported a highly significant association between focal loss of CD44v3 and CD44v6 immunostaining and short recurrence-free interval in noninvasive (pTa) urothelial carcinoma but not in minimally invasive carcinomas. These results suggest that the expression of CD44 is associated with differentiation and prognosis in bladder cancer. Whether the altered CD44 protein expression and tumor progression in urinary bladder cancer are causally related is still unclear. It is known that CD44 plays a definite role in cell-cell and cellmatrix interactions. Thus, its down-regulation would facilitate loss of cell-cell cohesion, detachment from the basement membrane, and subsequent infiltration of the underlying tissues.

We identified two patterns of loss of CD44 expression. One is seen in the background of normal staining pattern (i.e., CD44 expression in basal cells only), whereas the other is seen in urothelium with accentuated CD44 expression. Although we did not find any significant difference between the two patterns with respect to tumor grade, stage, or clinical outcome, these findings tempt one to hypothesize that the staining patterns may be reflective of a different underlying molecular pathogenetic pathway.

The regeneration of urothelium after urothelial damage is accompanied by changes in protein synthesis related to differentiation by mechanisms that have not yet been established (47). This transition from basal to terminally differentiated superficial cells in the urothelium is reflected in the morphological and antigenic characteristic of each layer. Cytokeratins are widely used as biochemical markers of epithelial differentiation (25). Our findings concur with those of others who have demonstrated that in normal urothelium, CK20 is occasionally expressed only in terminally differentiated umbrella cells. However, we and others $(20,24)$ observed that CK20 showed stronger and consistent IR in high-grade, less differentiated tumors. The 
TABLE 4. Comparison of Clinical Outcome with Stage, CK20, and CD44 IR in pTa and pT1 Papillary Urothelial Neoplasia

\begin{tabular}{|c|c|c|c|c|c|c|c|}
\hline & \multirow{2}{*}{ Total } & \multicolumn{2}{|c|}{ Stage } & \multicolumn{2}{|c|}{ CK20 IR } & \multicolumn{2}{|c|}{ CD44 IR } \\
\hline & & pTa & pT1 & negative & positive & N/Ac & Loss \\
\hline Recurrence* & $54.7 \%(58)$ & $56.4 \%$ & $50 \%$ & $44.9 \%$ & $63.2 \%$ & $40 \%$ & $63.6 \%$ \\
\hline$P$-value & & \multicolumn{2}{|c|}{0.559} & \multicolumn{2}{|c|}{0.06} & \multicolumn{2}{|c|}{0.18} \\
\hline Progression in stage ${ }^{* *}$ & $17.3 \%(17)$ & $12.1 \%$ & $21.1 \%$ & $15.2 \%$ & $19.2 \%$ & $10.5 \%$ & $21.7 \%$ \\
\hline$P$-value & & \multicolumn{2}{|c|}{0.325} & \multicolumn{2}{|c|}{0.601} & \multicolumn{2}{|c|}{0.156} \\
\hline
\end{tabular}

* Data available for tumor recurrence in 106 of 120 cases.

** Data available for tumor progression in stage in 98 of 120 cases.
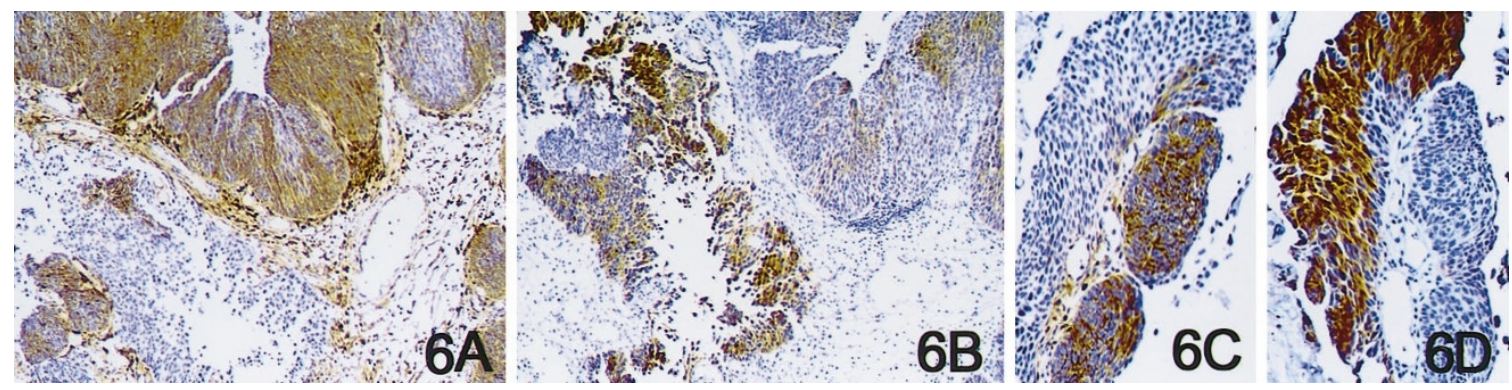

FIGURE 6. Inverse relationship of CK20 and CD44 immunoreactivity: areas showing CK20 positivity show loss of CD44 immunoreactivity and vice versa. (A, CD44; B, CK20; C, CD44; D, CK20).

TABLE 5. Relationship of CD44 and CK20 IR in pTa and pT1 Papillary Urothelial Neoplasia

\begin{tabular}{lcc}
\hline & CK20-negative & CK20-positive \\
\hline CD44 N/Ac & $71.5 \%$ & $4.6 \%$ \\
CD44 loss & $28.5 \%$ & $95.4 \%$ \\
$P$-value & & $\mathbf{< 0 . 0 0 1}$ \\
\hline
\end{tabular}

reasons for this apparent contradicting phenomenon are at this time unknown.

In contrast to CD44, which shows intense membrane staining only of the basal cell layers in normal urothelium, CK20 IR is seen in the terminally differentiated, superficial umbrella cells. We studied whether this inverse staining pattern of both molecules was also maintained in neoplastic lesions. We observed an inverse relationship between CK20 and CD44 within individual neoplasms. Although the molecular relationship between both markers has not yet been studied, it is known that the CD44 cytoplasmic tail interacts with underlying cytoskeleton proteins such as actin, ankyrin, or members of the ezrin-radixin-moesin family (48). The prominent changes in both CD44 and CK20 gene expression in papillary urothelial neoplasia might indicate significant cellular and molecular events leading to increasing tumor grade and progression in stage. Thus, further study into the interactions between CD44 and CK20 at the molecular level may give crucial information regarding the underlying pathobiologic process of superficial urothelial neoplasia.

Recently, several reports have suggested the use of a reverse transcription-polymerase chain reac- tion technique for the detection of CK20 expression in exfoliated urine cells as a useful, noninvasive diagnostic test for urothelial carcinoma and premalignant urothelial lesions $(23,24)$. As indicated by Southgate et al. (49), these studies were based on previous reports showing negative CK20 expression in urothelial cells grown in vitro, and it is known that urothelial cells grown in cultures fail to achieve terminal cytodifferentiation and thus do not express CK20. We further caution against the use of CK20 detection in urine cytology specimens as the sole screening method for bladder neoplasia because CK20 IR was totally absent in the neoplastic cells in $30.6 \%$ of high-grade papillary urothelial carcinomas.

In summary, CD44 and CK20 are significantly related to WHO/ISUP grade and to each other and have potential combined utility in predicting prognosis and behavior in pTa and pT1 papillary urothelial neoplasms. Further investigation of the relationship between CD44 and CK20 may shed further light on the molecular pathways involved in urinary bladder neoplasia.

Acknowledgments: Dr. Sangeeta Desai, pathologist, Tata Memorial Hospital, India, was awarded an International Cancer Research Transfer Technology Fellowship by the UICC in January 1999 to work on this project.

\section{REFERENCES}

1. Haukaas S, Daehlin L, Maartman-Moe H, Ulvik NM. The long-term outcome in patients with superficial transitional 
cell carcinoma of the bladder: a single-institutional experience. Br J Urol 1999;83:957-63.

2. Torti FM, Lum BL, Aston D, Mackenzie N, Faysel M, Shortliffe LD, Freiha F. Superficial bladder cancer: the primacy of grade in the development of invasive disease. J Clin Oncol 1987;5:125-30.

3. Heney NM, Ahmed S, Flanagan MJ, Frable W, Corder MP, Hafermann MD. Superficial bladder cancer: progression and recurrence. J Urol 1983;130:1083-6.

4. Malmstrom PU, Busch C, Norlen BJ. Recurrence, progression and survival in bladder cancer. Scand J Urol Nephrol 1987;21:185-95.

5. Kern WH. The grade and pathologic stage of bladder cancer. Cancer 1984;53:1185-9.

6. Loening S, Narayana A, Yoder L, Slymen D, Weinstein S, Penick G, Culp D. Factors influencing the recurrence rate of bladder cancer. J Urol 1980;123:29-31.

7. Epstein JI, Amin MB, Reuter VR, Mostofi FK. The World Health Organization/International Society of Urological Pathology consensus classification of urothelial (transitional cell) neoplasms of the urinary bladder. Bladder Consensus Conference Committee. Am J Surg Pathol 1998;22:1435-48.

8. Knowles MA. Molecular genetics of bladder cancer. Br J Urol 1995;75:57-66.

9. Nakanishi K, Kawai T, Suzuki M, Torikata C. Growth factors and oncogene products in transitional cell carcinoma. Mod Pathol 1996;9:292-7.

10. Abdel-Fattah R, Challen C, Griffiths TR, Robinson MC, Neal DE, Lunec J. Alterations of TP53 in microdissected transitional cell carcinoma of the human urinary bladder: high frequency of TP53 accumulation in the absence of detected mutations is associated with poor prognosis. Br J Cancer 1998;77:2230-8.

11. Korkolopoulou P, Christodoulou P, Kapralos P, Exarchakos M, Bisbiroula A, Hadjiyannakis $\mathrm{M}$, et al. The role of p53, MDM2 and c-erb B-2 oncoproteins, epidermal growth factor receptor and proliferation markers in the prognosis of urinary bladder cancer. Pathol Res Pract 1997;193:767-75.

12. Neal DE, Marsh C, Bennett MK, Abel PD, Hall RR, Sainsbury $\mathrm{JR}$, et al. Epidermal-growth-factor receptors in human bladder cancer: comparison of invasive and superficial tumours. Lancet 1985;1:366-8.

13. Wright C, Mellon K, Johnston P, Lane DP, Harris AL, Horne $\mathrm{CH}$, et al. Expression of mutant p53, c-erbB-2 and the epidermal growth factor receptor in transitional cell carcinoma of the human urinary bladder. Br J Cancer 1991;63:967-70.

14. Nguyen PL, Swanson PE, Jaszcz W, Aeppli DM, Zhang G, Singleton TP, et al. Expression of epidermal growth factor receptor in invasive transitional cell carcinoma of the urinary bladder. A multivariate survival analysis. Am J Clin Pathol 1994;101:166-76.

15. Lee SE, Chow NH, Chi YC, Tzai TS, Yang WH, Lin SN. Expression of c-erbB-2 protein in normal and neoplastic urothelium: lack of adverse prognostic effect in human urinary bladder cancer. Anticancer Res 1994;14:1317-24.

16. Ross JS, del Rosario AD, Bui HX, Kallakury BV, Okby NT, Figge J. Expression of the CD44 cell adhesion molecule in urinary bladder transitional cell carcinoma. Mod Pathol 1996;9:854-60.

17. Lipponen P, Aaltoma S, Kosma VM, Ala-Opas M, Eskelinen M. Expression of CD44 standard and variant -v6 proteins in transitional cell bladder tumors and their relation to prognosis during a long-term follow-up. J Pathol 1998;186:15764.

18. Toma V, Hauri D, Schmid U, Ackermann D, Maurer R, Alund $\mathrm{G}$, et al. Focal loss of CD44 variant protein expression is related to recurrence in superficial bladder carcinoma. Am J Pathol 1999;155:1427-32.
19. Harnden P, Allam A, Joyce AD, Patel A, Selby P, Southgate J. Cytokeratin 20 expression by non-invasive transitional cell carcinomas: potential for distinguishing recurrent from nonrecurrent disease. Histopathology 1995;27:169-74.

20. Harnden P, Eardley I, Joyce AD, Southgate J. Cytokeratin 20 as an objective marker of urothelial dysplasia. Br J Urol 1996;78:870-5.

21. Harnden P, Mahmood N, Southgate J. Expression of cytokeratin 20 redefines urothelial papillomas of the bladder. Lancet 1999;353:974-7.

22. Matsumura Y, Sugiyama M, Matsumura S, Hayle AJ, Robinson P, Smith JC, et al. Unusual retention of introns in CD44 gene transcripts in bladder cancer provides new diagnostic and clinical oncological opportunities. J Pathol 1995;77:1120.

23. Klein A, Zemer R, Buchumensky V, Klaper R, Nissenkorn I. Expression of cytokeratin 20 in urinary cytology of patients with bladder carcinoma. Cancer 1998;82:349-54.

24. Buchumensky V, Klein A, Zemer R, Kessler OJ, Zimlichman S, Nissenkorn I. Cytokeratin 20: a new marker for early detection of bladder cell carcinoma? J Urol 1998;160:1971-4.

25. Moll R, Lowe A, Laufer J, Franke WW. Cytokeratin 20 in human carcinomas. A new histodiagnostic marker detected by monoclonal antibodies. Am J Pathol 1992;140:427-47.

26. Moll I, Kuhn C, Moll R. Cytokeratin 20 is a general marker of cutaneous Merkel cells while certain neuronal proteins are absent. J Invest Dermatol 1995;104:910-5.

27. Liao HX, Lee DM, Levesque MC, Haynes BF. $N$-terminal and central regions of the human CD44 extracellular domain participate in cell surface hyaluronan binding. J Immunol 1995;155:3938-45.

28. Bourguignon LY, Zhu H, Shao L, Zhu D, Chen YW. Rhokinase (ROK) promotes $\mathrm{CD} 44 \mathrm{v}(3,8-10)$-ankyrin interaction and tumor cell migration in metastatic breast cancer cells. Cell Motil Cytoskel 1999;43:269-87.

29. Rudzki Z, Jothy S. CD44 and the adhesion of neoplastic cells. Mol Pathol 1997;50:57-71.

30. Bourguignon LY, Gunja-Smith Z, Iida N, Zhu HB, Young LJ, Muller WJ, et al. CD44v(3,8-10) is involved in cytoskeletonmediated tumor cell migration and matrix metalloproteinase (MMP-9) association in metastatic breast cancer cells. J Cell Physiol 1998;176:206-15.

31. Borland G, Ross JA, Guy K. Forms and functions of CD44. Immunology 1998;93:139-48.

32. Charrad RS, Li Y, Delpech B, Balitrand N, Clay D, Jasmin C, et al. Ligation of the CD44 adhesion molecule reverses blockage of differentiation in human acute myeloid leukemia. Nat Med 1999;5:669-76.

33. Yu Q, Stamenkovic I. Localization of matrix metalloproteinase 9 to the cell surface provides a mechanism for CD44mediated tumor invasion. Genes Dev 1999;13:35-48.

34. Joensuu H, Klemi PJ, Toikkanen S, Jalkanen S. Glycoprotein CD44 expression and its association with survival in breast cancer. Am J Pathol 1993;143:867-74.

35. Washington K, Gottfried MR, Telen MJ. Expression of the cell adhesion molecule CD44 in gastric adenocarcinomas. Hum Pathol 1994;25:1043-9.

36. Coppola D, Hyacinthe M, Fu L, Cantor AB, Karl R, Marcet J, et al. CD44V6 expression in human colorectal carcinoma. Hum Pathol 1998;29:627-35.

37. Wielenga VJ, van der Neut R, Offerhaus GJ, Pals ST. CD44 glycoproteins in colorectal cancer: expression, function, and prognostic value. Adv Cancer Res 2000;77:169-87.

38. American Joint Committee on Cancer. Urinary bladder. In AJCC cancer staging manual. 5th ed. Philadelphia: Lippincott-Raven; 1997. p. 241-3.

39. Sugino T, Gorham H, Yoshida K, Bolodeoku J, Nargund V, Cranston D, et al. Progressive loss of CD44 gene expression in invasive bladder cancer. Am J Pathol 1996;149:873-82. 
40. Holmang S, Hedelin H, Anderstrom C, Holmberg E, Busch C, Johnasson SL. Recurrence and progression in low grade papillary urothelial tumors. J Urol 1999;162:702-7.

41. Cheng L, Darson M, Chevill JC, Neumann RM, Zincke H, Nehra A, et al. Urothelial papilloma of the bladder. Clinical and biologic implications. Cancer 1999;86:2098-101.

42. Cheng L, Neumann RM, Bostwick DG. Papillary urothelial neoplasms of low malignant potential. Clinical and biologic implications. Cancer 1999;86:2102-8.

43. Cheng L, Neumann RM, Nehra A, Spotts BE, Weaver AL, Bostwick DG. Cancer heterogeneity and its biologic implications in the grading of urothelial carcinoma. Cancer 2000;88:1663-70.

44. Alho AM, Underhill CB. The hyaluronate receptor is preferentially expressed on proliferating epithelial cells. J Cell Biol 1989;108:1557-65.

45. McKenny JK, Desai S, Cohen C, Amin MB. Discriminatory immunohistochemical staining of urothelial carcinoma in situ (CIS) and non-neoplastic urothelium: an analysis of CK20, p53, and CD44 antigens [abstract]. Mod Pathol 2000; 113:107A.

46. Sugino T, Yoshida K, Zhao S, Goodison S, Tarin D. Disorderly CD44 gene expression in human cancer cells can be modulated by growth conditions. J Pathol 1998;186:17-23.

47. Romih R, Jezernik K, Masera A. Uroplakins and cytokeratins in the regenerating rat urothelium after sodium saccharin treatment. Histochem Cell Biol 1998;109:263-9.

48. Lokeshwar VB, Bourguignon LY. Post-translational protein modification and expression of ankyrin-binding site(s) in GP85 (Pgp-1/CD44) and its biosynthetic precursors during T-lymphoma membrane biosynthesis. J Biol Chem 1991;266: 17983-9.

49. Southgate J, Lobban D, Harnden P. Expression of cytokeratin 20 in urinary cytology of patients with bladder carcinoma [letter]. Cancer 1998;83:1052-4.

\section{Book Review}

McLendon RE, Bigner DD, Bigner SH, Provenzale JM: Pathology of Tumors of the Central Nervous System. A Guide to Histologic Diagnosis, 313 pp, London, Arnold, 2000 (\$179.50).

This book has been designed as a practical diagnostic manual and atlas and serves as a companion text to the sixth edition of Russell and Rubinstein's Pathology of Tumors of the Nervous System. For this endeavor, two of the editors of Russell and Rubinstein have been joined by the pathologist Sandra Bigner and the neuroradiologist James Provenzale.

The book is divided into three main sections: mass lesions in adult brains, mass lesions in brains of children and young adults, and brain tumors of maldevelopmental origin. Given this approach, a certain degree of overlap is inevitable, and this is reflected in the discussion of glioblastoma. However, in considering the differential diagnosis for the different age groups, this approach works very well. Most entities have a highlighted table of the salient epidemiological and pathological features. There are also very useful tables for mimics of fibrillary astrocytoma, melanocytic lesions, and cystic lesions of the central nervous system. Newer entities such as the papillary glioneuronal tumor, atypical teratoid tu- mor, solitary fibrous tumor of the meninges, and the desmoplastic infantile tumors (ganglioglioma and astrocytoma) are also included.

The discussion stresses features of practical diagnostic importance, the use of immunohistochemical markers, and the value of Mib 1 labeling in assessing proliferative potential. The molecular biology of brain tumors is not addressed, and this appears entirely justified given the practical diagnostic bias of the book. The text is well organized and packs in considerable detail for an atlas format and also includes selected references. The discussion pertaining to high-grade astrocytomas of children and young adults is particularly good and highlights the problems in differential diagnosis in this group.

The book is generously illustrated with highquality photographs of gross and microscopic pathology, and neuro-imaging illustrations greatly enhance the value of this atlas. Given its practical bias, this book is a valuable addition to the standard texts on pathology of central nervous system tumors. In many ways, it is likely even more useful as a 'bench' text for pathologists in the diagnosis of brain tumors.

\section{M.B. Bhattacharjee \\ Tulane University Medical School \\ New Orleans, Louisiana}

